\title{
Sound Radiation from an Elastically Restrained Plate Covered by an Acoustic Decoupling Layer
}

\author{
Gang Wang, ${ }^{1}$ Wan-You Li, ${ }^{1}$ Wen Long $\mathrm{Li}^{2}{ }^{2}$ and Jingtao $\mathrm{Du}^{1}$ \\ ${ }^{1}$ College of Power and Energy Engineering, Harbin Engineering University, Harbin 150001, China \\ ${ }^{2}$ Advanced Engineering and Technologies, 8446 Chatham Drive, Canton, MI 48187, USA \\ Correspondence should be addressed to Wan-You Li; hrbeu_ripet_lwy@163.com
}

Received 1 September 2015; Revised 27 October 2015; Accepted 5 November 2015

Academic Editor: George Tsiatas

Copyright (c) 2015 Gang Wang et al. This is an open access article distributed under the Creative Commons Attribution License, which permits unrestricted use, distribution, and reproduction in any medium, provided the original work is properly cited.

\begin{abstract}
The sound radiation from elastically restrained plates covered by a decoupling layer is studied using the Spectrogeometric Method (SGM), which is a meshless and parametric modeling technique. By adopting the Rayleigh-Ritz procedure and the Rayleigh integral, a vibroacoustic coupling system is established. This model studies the situation when the plate is immersed in heavy fluid, such as water, in which the strong coupling between the structure and sound field should be fully considered. The influence of the boundary conditions on the radiated sound power and sound reduction provided by the decoupling layer based on the locally reacting model is studied. The nonuniform distributed decoupling layer is also studied to analyze the sound reduction effect. The sound intensity on the outer surface of the decoupling layer is investigated and tends to be uniform along the plate scale with increasing thickness of the decoupling layer.
\end{abstract}

\section{Introduction}

The addition of decoupling materials to an elastic vibration structure is a significant approach to reduce the sound radiated from the structure immersed in heavy fluid. The decoupling materials should have a low stiffness in comparison to the base structure to support thickness deformation induced by the action of dynamic pressure in the fluid. This deformation enables isolation of the fluid from the vibration of the base structure.

Sound radiation from plates in a heavy fluid has been fully studied by taking the fluid-structure coupling into consideration [1-8]. To reduce the sound radiation, the addition of decoupling materials to the surface of the plates has been studied in recent years. In the literature, there are mainly two models to analyze the decoupling layer: the three-dimensional theory of elasticity [9-15] and the locally reacting model [16-21]. The former model accounts for thickness deformation, bending, shear, and extensional deformation of the decoupling layer. The latter model, the locally reacting model, assumes that the decoupling layer behaves as evenly distributed massless springs on the base structure. Chonan and Kugo [9] investigated the theoretical coincidence frequency and transmission characteristics of two-layer infinite plates excited by a plane acoustic wave with the use of the Lame potential function. Ko [10] developed a theoretical model to evaluate the reduction of structureborne noise from an infinite plate coated with an air-voided elastomeric baffle. A lighter material is found to be more suitable for the reduction of structure-borne noise. Accounting for the attached distributed inhomogeneity, Zhang and Pan $[12,13]$ established a theoretical model to investigate the sound radiation and absorption properties of coated infinite plates. Tao et al. [14] developed a vibroacoustic coupled model to predict the noise reduction performance of a void decoupling layer covered on an infinite bare plate based on the impedance transfer matrix. To simplify the formula of sound radiation from the decoupling layer, the locally reacting model is commonly used, especially when the density of the decoupling is small, and therefore is more suitable for the sound reduction [10]. Laulagnet and Guyader $[18,20]$ used the locally reacting model to calculate the sound radiated from a thin, simply supported cylindrical shell covered by a compliant coating. This method was compared 
to a rigorous model that involves the three-dimensional theory of elasticity for the compliant layer motion, and the results revealed that the locally reacting model appropriately describes the vibroacoustic behavior of thin shells covered by a decoupling layer. Sandman and Boisvert [19] used the locally reacting model in the case that the base structure is a rigid piston. Foin et al. [17] studied the sound radiation from a simply supported rectangular plate using the locally reacting model. Xiao et al. [22] present an experimental investigation into the dynamic response of three free floating stiffened metal boxes with protective coatings subjected to underwater explosion. Sanches et al. [23] developed a direct version of the boundary element method to model the stationary dynamic response of reinforced plate structures to realize the noise or vibration reduction. The free vibration of sandwich panels was studied by Brischetto et al. [24] by introducing the zigzag function in the displacement models of classical and higher-order two-dimensional shell theories. Functionally graded material panels [25] were studied using generalized differential quadrature method to determine the static and dynamic responses.

Previous work mainly focused on the classic mounting conditions of the plates, especially with the simply supported ones. The mounting conditions of a vibrating plate are known to have significant influence on its sound radiation [26, 27]. The radiation from elastically mounted plates has been studied recently [28-30]. To the author's knowledge, there is no work focusing on the radiation from an elastically restrained plate with a decoupling layer on it. In the literature, the decoupling layer is studied when it is uniformly distributed on the surface. Motivated by a proposal for attaching a finite signal conditioning plate (SCP), Zhang and Pan $[12,13]$ studied the sound reduction of a plate covered by decoupling layer and an SCP as a distributed discontinuity. For a plate of large scale, the thickness of the decoupling layer on the plate may be nonuniformly distributed to obtain different sound reduction effects at different positions of the plate.

The present paper aims to develop an exact model for the sound radiation characteristics from elastically mounted plates covered by a decoupling acoustic coating layer. The plate is elastically restrained against both translation and rotation along the edges. By adopting the Fourier series expansion, a nonuniform distributed decoupling layer is also studied. The plate displacement function is universally expressed as a two-dimensional Fourier cosine series supplemented with several one-dimensional series that are introduced to address the potential convergence difficulties. Both the displacement function of the outer surface of decoupling layer and the sound pressure radiated from the decoupling layer are expressed as the combination of a Fourier cosine series. By adopting the Rayleigh-Ritz procedure and the Rayleigh integral, the strong coupling between the structure and acoustic space is considered. Classic and elastically mounted boundaries can be realized by setting the restrained springs at boundaries to infinity or to a given value. The proposed formulation helps to reduce the computational time by converting the quadruple integral into four single integrals via Fourier expansion. The analytical results are validated with those in the literature. The effect of restrained stiffness and the thickness of the decoupling layer on the sound reduction is studied.

\section{Theory}

A typical configuration of the vibroacoustic coupling model of an elastically restrained plate backed in an infinite rigid baffle is shown in Figure 1. The plate is covered with a decoupling layer on the acoustic-space side. The Love-Kirchhoff plate theory is adopted, which only considers the transverse bending deformation. The decoupling layer is described as the locally reacting model. The plate-baffled system separates a heavy fluid from vacuum with a point force applied on the base plate. Considering the deformation of the decoupling layer, the displacements of the base plate and the outer surface of decoupling layer are $w_{1}$ and $w_{2}$, respectively.

For the rectangular plate with general elastic boundary supports, its flexural displacement can be invariantly expanded into a modified Fourier series as [23]

$$
\begin{aligned}
& w_{1}(x, y)=\sum_{m=0}^{M} \sum_{n=0}^{N} A_{m n} \cos \lambda_{a m} x \cos \lambda_{b n} y \\
& +\sum_{j=1}^{4}\left(\xi_{b}^{j}(y) \sum_{m=0}^{M} c_{m}^{j} \cos \lambda_{a m} x\right. \\
& \left.+\xi_{a}^{j}(x) \sum_{n=0}^{N} d_{n}^{j} \cos \lambda_{b n} y\right),
\end{aligned}
$$

where $\lambda_{a m}=m \pi / a, \lambda_{b n}=n \pi / b$, and $A_{m n}, c_{m}^{j}$, and $d_{n}^{j}$ represent the unknown Fourier expansion coefficients to be determined. The supplementary functions $\xi_{b}^{j}(y)$ and $\xi_{a}^{j}(x)$ are introduced to overcome the potential discontinuities encountered when the displacement function and its lowerorder derivatives are periodically extended onto the entire $x-$ $y$ plane, as mathematically implied by the Fourier expansion. As an immediate numerical benefit, the Fourier series in (1) will converge uniformly at an accelerated rate.

For the decoupling layer, the displacement of the outer surface is expressed as a standard Fourier cosine series:

$$
w_{2}(x, y)=\sum_{m=0}^{M} \sum_{n=0}^{N} B_{m n} \cos \lambda_{a m} x \cos \lambda_{b n} y,
$$

in which $B_{m n}$ represents the Fourier coefficients for the displacement of the outer surface. The sound pressure on the outer surface of the decoupling layer is simply expressed as a standard Fourier cosine series:

$$
p(x, y)=\sum_{m=0}^{M} \sum_{n=0}^{N} P_{m n} \cos \lambda_{a m} x \cos \lambda_{b n} y,
$$

where $P_{m n}$ denotes the complex Fourier coefficients for the pressure of the sound field.

The decoupling layer is considered as a locally reacting decoupling layer, in which it is assumed that the deformation of the decoupling layer depends on the surface acoustic 


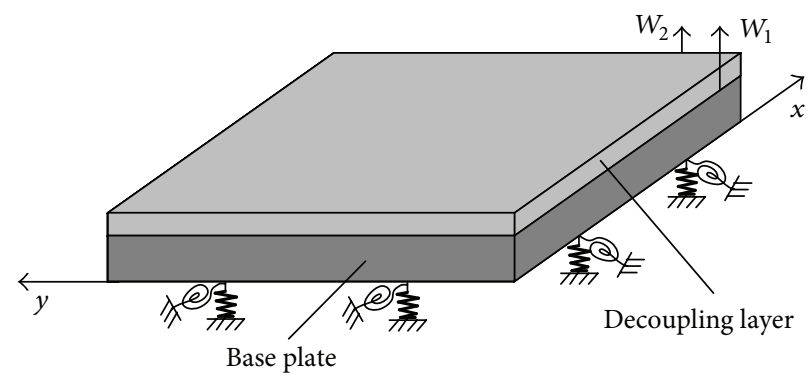

FIGURE 1: Schematic illustration of sound radiated form an elastically restrained plate covered by a decoupling layer.

pressure and the impedance of the decoupling layer. The deformation of the decoupling layer is described by the following relation:

$$
p(x, y)=\left(w_{1}(x, y)-w_{2}(x, y)\right) Z(x, y),
$$

in which $Z(x, y)$ is the impedance of the decoupling layer. Based on the locally reacting model, $Z(x, y)$ could be expressed as

$$
Z(x, y)=\frac{B_{c}\left(1+\eta_{c}\right)}{h_{c}(x, y)},
$$

in which $B_{c}, \eta_{c}$, and $h_{c}(x, y)$ are, respectively, the modulus of the decoupling layer, the damping modulus, and the thickness of the decoupling layer on the plate. Assuming that the decoupling layer acts as a massless spring, the stress acting on the base plate is the same as the surface acoustic pressure $p(x, y)$ on the decoupling layer. In this paper, the decoupling layers with varying thickness along the plate scale are taken into consideration. To account for the arbitrariness of the decoupling layer distribution, the impedance of the coupling layer is expanded into a Fourier cosine series:

$$
\begin{aligned}
Z(x, y) & =\frac{B_{c}\left(1+\eta_{c}\right)}{h_{c}(x, y)} \\
& =\sum_{m=0}^{M} \sum_{n=0}^{M} Z_{m n} \cos \lambda_{a m} x \cos \lambda_{b n} y .
\end{aligned}
$$

Once suitable forms of solutions have been chosen, the Rayleigh-Ritz procedure can be used to calculate the unknown expansion coefficients. The Lagrangian for the plate structure can be written as

$$
L_{\text {plate }}=U_{\text {plate }}-T_{\text {plate }}+W_{p}-W_{F},
$$

where $U_{\text {plate }}$ is the total potential energy associated with the transverse deformation of the plate and the deflections of the restraining springs; $T_{\text {plate }}$ denotes the total kinetic energy of the plate; $W_{F}$ represents the mechanical work introduced by the exciting force acting on the base plate; and $W_{p}$ is the mechanical work introduced by the normal stress acting on the base plate, which is written as

$$
W_{p}=\int_{0}^{b} \int_{0}^{a} w_{1}(x, y) p(x, y) d x d y .
$$

The total potential and kinetic energy for the plate can be explicitly expressed as

$$
\begin{aligned}
& U_{\text {plate }}=\frac{D}{2} \int_{0}^{b} \int_{0}^{a}\left\{\left(\frac{\partial^{2} w_{1}}{\partial x^{2}}\right)^{2}+\left(\frac{\partial^{2} w_{1}}{\partial y^{2}}\right)^{2}\right. \\
& \left.+2 \mu \frac{\partial^{2} w_{1}}{\partial x^{2}} \frac{\partial^{2} w_{1}}{\partial y^{2}}+2(1-\mu)\left(\frac{\partial^{2} w_{1}}{\partial x \partial y}\right)^{2}\right\} d x d y \\
& +\frac{1}{2} \int_{0}^{b}\left[k_{x 0} w_{1}^{2}+K_{x 0}\left(\frac{\partial w_{1}}{\partial x}\right)^{2}\right]_{x=0} d y+\frac{1}{2} \\
& \cdot \int_{0}^{b}\left[k_{x a} w_{1}^{2}+K_{x a}\left(\frac{\partial w_{1}}{\partial x}\right)^{2}\right]_{x=a} d y+\frac{1}{2} \\
& \cdot \int_{0}^{a}\left[k_{y 0} w_{1}^{2}+K_{y 0}\left(\frac{\partial w_{1}}{\partial y}\right)^{2}\right]_{y=0} d x+\frac{1}{2} \\
& \cdot \int_{0}^{a}\left[k_{y b} w_{1}^{2}+K_{y b}\left(\frac{\partial w_{1}}{\partial y}\right)^{2}\right]_{y=b} d x, \\
& T_{\text {plate }}=\frac{1}{2} \rho h \omega^{2} \int_{0}^{b} \int_{0}^{a} w_{1} d x d y,
\end{aligned}
$$

in which $\omega, D, \mu, \rho$, and $h$ are the frequency of the exciting force in radians, the flexible rigidity, the Poisson ratio, the mass density, and the thickness of the plate, respectively, and $k_{x 0}$ and $K_{x 0}$ are the translational and rotational stiffness restrained at $x=0$, respectively, which are also applicable for $k_{x a}, k_{y 0}, k_{y b}, K_{x a}, K_{y 0}$, and $K_{y b}$. To account for the arbitrariness of the stiffness distribution for each elastic restraint, the stiffness function is expanded into a simple Fourier cosine series; for example,

$$
k_{x 0}(y)=\sum_{l=0}^{\infty} \widetilde{k}_{x 0, l} \cos \left(\frac{l \pi}{b} y\right),
$$

where $\widetilde{k}_{x 0, l}$ is the Fourier coefficients of the Fourier cosine series expansion of the stiffness function $k_{x 0}(y)$ along $x=$ 0 . It can be proved mathematically that the cosine series expansion of any continuous stiffness function converges at a rate of at least $(l \pi)^{2}$. 
The work done by the point force (acting on the base plate) can be calculated as

$$
\begin{aligned}
W_{F} & =\int_{0}^{b} \int_{0}^{a} w_{1}(x, y) F(x, y) \delta\left(x-x_{0}\right) \\
& \delta \delta\left(y-y_{0}\right) d x d y
\end{aligned}
$$

in which $\delta$ is the Dirac function, $F(x, y)$ is a harmonic point force with the frequency of $\omega$ in radians, and $\left(x_{0}, y_{0}\right)$ is the location where the force is applied.

Substituting (8), (9), and (11) into (7) and then minimizing (7) against the unknown Fourier coefficients result in the following:

$$
\left.\left\{\begin{array}{ll}
\mathbf{K}_{p} & \mathbf{C}
\end{array}\right]-\omega^{2}\left[\begin{array}{ll}
\mathbf{M}_{p} & \mathbf{0}
\end{array}\right]\right\}\left\{\begin{array}{l}
\mathbf{A} \\
\mathbf{P}
\end{array}\right\}=\left\{\begin{array}{l}
\mathbf{F} \\
\mathbf{0}
\end{array}\right\}
$$

in which $\mathbf{A}$ and $\mathbf{P}$ are the Fourier coefficients in (1) and (3) and $\mathbf{C}, \mathbf{K}_{p}$, and $\mathbf{M}_{p}$ are the coupling matrix, the stiffness matrix, and the mass matrix of the plate, respectively.

Because the thickness variation of the decoupling layer is comparatively small compared to the acoustic wavelength in water when the frequency is lower than $3000 \mathrm{~Hz}$, we consider in this paper that the acoustic pressure acting on the outer surface of the decoupling layer could be obtained by the Rayleigh integral:

$$
p(x, y)=-\frac{i \omega \rho_{0}}{2 \pi} \int_{0}^{b} \int_{0}^{a} \frac{\dot{w}_{2}\left(x^{\prime}, y^{\prime}\right) e^{i k R}}{R} d x^{\prime} d y^{\prime}
$$

where $k$ is the acoustic wave number, $\rho_{0}$ is the density of the acoustic medium, $R=\sqrt{\left(x-x^{\prime}\right)^{2}+\left(y-y^{\prime}\right)^{2}}$, and $\dot{w}_{2}$ is the velocity of the outer surface of the decoupling layer, $\dot{w}_{2}=i \omega w_{2}$. To effectively calculate the integral in (13), the integrand will be first expanded into a Fourier cosine series as

$$
\begin{aligned}
& \frac{e^{i k R}}{R}=\frac{e^{i k \sqrt{\tau^{2}+\sigma^{2}}}}{\sqrt{\tau^{2}+\sigma^{2}}}=\sum_{t=0}^{T} \sum_{s=0}^{S} E_{t s} \cos \left(\lambda_{a t} \tau\right) \cos \left(\lambda_{b s} \sigma\right) \\
& =\sum_{t=0}^{T} \sum_{s=0}^{S} E_{t s}\left(\cos \lambda_{a t} x \cos \lambda_{a t} x^{\prime} \cos \lambda_{b s} y \cos \lambda_{b s} y^{\prime}\right. \\
& +\cos \lambda_{a t} x \cos \lambda_{a t} x^{\prime} \sin \lambda_{b s} y \sin \lambda_{b s} y^{\prime} \\
& +\sin \lambda_{a t} x \sin \lambda_{a t} x^{\prime} \cos \lambda_{b s} y \cos \lambda_{b s} y^{\prime} \\
& \left.+\sin \lambda_{a t} x \sin \lambda_{a t} x^{\prime} \sin \lambda_{b s} y \sin \lambda_{b s} y^{\prime}\right),
\end{aligned}
$$

where $\tau=x-x^{\prime}, \sigma=y-y^{\prime}$, and $E_{t s}$ are Fourier expansion coefficients. In the implementations, the Fourier coefficients $E_{t s}$ are actually calculated using a Discrete Cosine Transform (DCT) or FFT.
Substituting (2), (3), and (14) into (13) and making use of the orthogonality of the cosine functions yields

$$
\begin{gathered}
\kappa_{m} \kappa_{n} P_{m n}=\frac{\omega^{2} \rho_{0}}{2 \pi a b} \sum_{m^{\prime}=0}^{M} \sum_{n^{\prime}=0}^{N} \sum_{t=0}^{T} \sum_{s=0}^{S} \int_{0}^{b} \int_{0}^{a} \int_{0}^{b} \int_{0}^{a} B_{m^{\prime} n^{\prime}} E_{t s} \\
\cdot\left(\cos \lambda_{a m} x \cos \lambda_{b n} y \cos \lambda_{a m^{\prime}} x^{\prime} \cos \lambda_{b n^{\prime}} y^{\prime} \cos \lambda_{a t} x\right. \\
\cdot \cos \lambda_{a t} x^{\prime} \cos \lambda_{b s} y \cos \lambda_{b s} y^{\prime}+\cos \lambda_{a m} x \cos \lambda_{b n} y \\
\cdot \cos \lambda_{a m^{\prime}} x^{\prime} \cos \lambda_{b n^{\prime}} y^{\prime} \cos \lambda_{a t} x \cos \lambda_{a t} x^{\prime} \sin \lambda_{b s} y \\
\cdot \sin \lambda_{b s} y^{\prime}+\cos \lambda_{a m} x \cos \lambda_{b n} y \cos \lambda_{a m^{\prime}} x^{\prime} \\
\cdot \cos \lambda_{b n^{\prime}} y^{\prime} \sin \lambda_{a t} x \sin \lambda_{a t} x^{\prime} \cos \lambda_{b s} y \cos \lambda_{b s} y^{\prime} \\
+\cos \lambda_{a m} x \cos \lambda_{b n} y \cos \lambda_{a m^{\prime}} x^{\prime} \cos \lambda_{b n^{\prime}} y^{\prime} \sin \lambda_{a t} x \\
\left.\cdot \sin \lambda_{a t} x^{\prime} \sin \lambda_{b s} y \sin \lambda_{b s} y^{\prime}\right) d x d y d x^{\prime} d y^{\prime},
\end{gathered}
$$

in which

$$
\kappa_{s}=\left\{\begin{array}{ll}
1 & s=0 \\
\frac{1}{2} & s \neq 0
\end{array} \quad(s=m, n) .\right.
$$

It is evident from (15) that the quadruple integrals have been reduced into a number of single integrals, which can all be readily calculated in a closed form.

Equation (15) can be rewritten as

$$
\boldsymbol{\kappa}\{\mathbf{P}\}=\omega^{2} \mathbf{Q}\{\mathbf{B}\},
$$

in which $\mathbf{B}, \boldsymbol{\kappa}$, and $\mathbf{Q}$ is the Fourier coefficients in (2), the matrix of $\kappa_{s}$, and the integral of cosine function in (15).

The normal displacement of the plate is given by (1). In accordance with the pressure expression, we will also reduce the displacement into a simple cosine series by simply expanding the supplementary functions, $\xi_{b}^{j}(y)$ and $\xi_{a}^{j}(x)$, into the Fourier cosine series. Thus, the displacement of the plate simplifies to

$$
\begin{aligned}
& w_{1}(x, y)=\sum_{m=0}^{M} \sum_{n=0}^{N} A_{m n} \cos \lambda_{a m} x \cos \lambda_{b n} y \\
& +\sum_{j=1}^{4}\left(\xi_{b}^{j}(y) \sum_{m=0}^{M} c_{m}^{j} \cos \lambda_{a m} x\right. \\
& \left.+\xi_{a}^{j}(x) \sum_{n=0}^{N} d_{n}^{j} \cos \lambda_{b n} y\right) \\
& =\sum_{m=0}^{M} \sum_{n=0}^{N} \bar{A}_{m n} \cos \lambda_{a m} x \cos \lambda_{b n} y
\end{aligned}
$$

in which

$$
\bar{A}_{m n}=A_{m n}+\sum_{l=1}^{4}\left(\widetilde{\beta}_{n}^{l} c_{m}^{l}+\widetilde{\alpha}_{m}^{l} d_{n}^{l}\right)
$$


where

$$
\begin{aligned}
& \xi_{a}^{l}(x)=\sum_{m=0}^{M} \widetilde{\alpha}_{m}^{l} \cos \lambda_{a m} x \\
& \xi_{b}^{l}(y)=\sum_{n=0}^{N} \widetilde{\beta}_{n}^{l} \cos \lambda_{b n} y .
\end{aligned}
$$

Equation (18) can be rewritten in matrix form as follows:

$$
\{\overline{\mathbf{A}}\}=\mathbf{T}\{\mathbf{A}\} .
$$

Substituting (1), (2), (3), and (6) into (4), one gets

$$
\begin{aligned}
\sum_{m=0}^{M} & \sum_{n=0}^{N} P_{m n} \cos \lambda_{a m} x \cos \lambda_{b n} y \\
= & \left(\sum_{m=0}^{M} \sum_{n=0}^{N} Z_{m n} \cos \lambda_{a m} x \cos \lambda_{b n} y\right) \\
& \cdot\left(\sum_{m=0}^{M} \sum_{n=0}^{N} \bar{A}_{m n} \cos \lambda_{a m} x \cos \lambda_{b n} y\right. \\
& \left.-\sum_{m=0}^{M} \sum_{n=0}^{N} B_{m n} \cos \lambda_{a m} x \cos \lambda_{b n} y\right)
\end{aligned}
$$

Making use of the orthogonality of the cosine functions, one is able to yield

$$
\boldsymbol{\kappa}\{\mathbf{P}\}=\mathbf{Z}_{A}\{\overline{\mathbf{A}}\}-\mathbf{Z}_{B}\{\mathbf{B}\}
$$

Combining (12), (17), and (23), the final system equation can be derived as

$$
\begin{aligned}
& \left\{\left[\begin{array}{ccc}
\mathbf{K}_{p} & \mathbf{0} & \mathbf{C} \\
\mathbf{Z}_{A} \mathbf{T} & -\mathbf{Z}_{B} & -\boldsymbol{\kappa} \\
\mathbf{0} & \mathbf{0} & \boldsymbol{\kappa}
\end{array}\right]-\omega^{2}\left[\begin{array}{ccc}
\mathbf{M}_{p} & \mathbf{0} & \mathbf{0} \\
\mathbf{0} & \mathbf{0} & \mathbf{0} \\
\mathbf{0} & \mathbf{Q} & \mathbf{0}
\end{array}\right]\right\}\left\{\begin{array}{l}
\mathbf{A} \\
\mathbf{B} \\
\mathbf{P}
\end{array}\right\} \\
& \quad=\left\{\begin{array}{l}
\mathbf{F} \\
\mathbf{0} \\
\mathbf{0}
\end{array}\right\} .
\end{aligned}
$$

Note that (24) represents a strongly coupled vibroacoustic system; that is, the interactions between the vibration of the plate and the pressure fields have been faithfully taken into account. Thus, the sound pressure distribution on the outer surface of the decoupling layer and the displacements of base plate and decoupling layer can be simultaneously obtained separately.

For the situation without decoupling layer, the relation between acoustic pressure and the displacements of base plate and decoupling layer, (4), is ignored. The Rayleigh integral is reduced to

$$
\boldsymbol{\kappa}\{\mathbf{P}\}=\omega^{2} \mathbf{Q}\{\overline{\mathbf{A}}\}
$$

Combining (12) and (25), we obtain the system equation without the decoupling layer:

$$
\left\{\left[\begin{array}{cc}
\mathbf{K}_{p} & \mathbf{C} \\
\mathbf{0} & \boldsymbol{\kappa}
\end{array}\right]-\omega^{2}\left[\begin{array}{ll}
\mathbf{M}_{p} & \mathbf{0} \\
\mathbf{Q T} & \mathbf{0}
\end{array}\right]\right\}\left\{\begin{array}{l}
\mathbf{A} \\
\mathbf{P}
\end{array}\right\}=\left\{\begin{array}{l}
\mathbf{F} \\
\mathbf{0}
\end{array}\right\} .
$$

By comparing the results obtained by (24) and (26), one could study the sound reduction provided by the decoupling layers.

In this paper, several indicators are introduced to analyze the vibration reduction provided by the decoupling layer. The indicators are the mean-square transverse velocity of the base plate, the radiated sound power into the fluid, the sound power reduction, and the sound intensity on the outer surface of decoupling layer. The mean-square transverse velocity of the base plate is averaged with respect to both time and space; namely,

$$
\begin{aligned}
\left\langle V_{1}^{2}\right\rangle & =\frac{1}{2 a b} \int_{0}^{b} \int_{0}^{a} \dot{w}_{1}(x, y) \dot{w}_{1}^{*}(x, y) d x d y \\
& =-\sum_{m=0}^{M} \sum_{n=0}^{N} \frac{\omega^{2}}{2} \kappa_{m} \kappa_{n} \bar{A}_{m n} \bar{A}_{m n}^{*}
\end{aligned}
$$

in which $*$ indicates the complex conjugate. The total radiated acoustic power from the decoupling layer can be calculated from

$$
\begin{aligned}
W_{2} & =\frac{1}{2} \operatorname{Re} \int_{0}^{b} \int_{0}^{a} p(x, y) \dot{w}_{2}^{*}(x, y) d x d y \\
& =\frac{a b}{2} \operatorname{Re} \sum_{m=0}^{M} \sum_{n=0}^{N} i \omega \kappa_{m} \kappa_{n} P_{m n} B_{m n}^{*}
\end{aligned}
$$

in which Re denotes the real part of a complex number. When there is no decoupling layer on the plate, the total radiated acoustic power from the base plate can be written as

$$
\begin{aligned}
W_{1} & =\frac{1}{2} \operatorname{Re} \int_{0}^{b} \int_{0}^{a} p(x, y) \dot{w}_{1}^{*}(x, y) d x d y \\
& =\frac{a b}{2} \operatorname{Re} \sum_{m=0}^{M} \sum_{n=0}^{N} i \omega \kappa_{m} \kappa_{n} P_{m n} \bar{A}_{m n}^{*} .
\end{aligned}
$$

The sound power reduction index is introduced to analyze the sound reduction effect of the decoupling layer, which is defined as

$$
\tau=10 \log _{10}\left(\frac{W_{1}}{W_{2}}\right) .
$$

The sound intensity on the outer surface of the decoupling layer can be obtained as

$$
I(x, y)=\operatorname{Re}\left(\dot{w}_{2}^{*}(x, y) p(x, y)\right) .
$$

In the following part, several cases are analyzed to study the radiated sound power and sound reduction provided by the decoupling layer. 


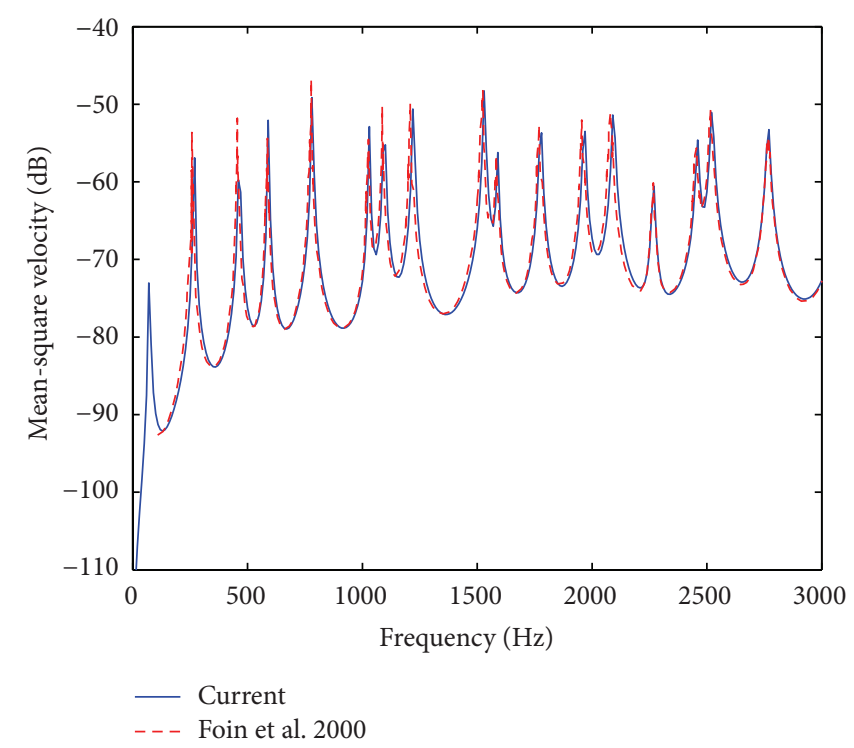

FIGURE 2: Mean-square velocity of the base plate compared with [17].

\section{Results}

In this section, the proposed formulation will first be validated against some existing results in the literature. The sound power radiation and reduction will be then presented for plates with various boundary conditions. In the following examples, the parameters are specified as $a \times b=0.6 \mathrm{~m} \times$ $0.6 \mathrm{~m}, \rho_{\text {plate }}=7850 \mathrm{~kg} / \mathrm{m}^{3}, E=2.1 \times 10^{11} \mathrm{~Pa}, h_{\text {plate }}=0.009 \mathrm{~m}$, and $v=0.3$ for the plate and as $\rho_{\text {water }}=1000 \mathrm{~kg} / \mathrm{m}^{3}$ and $c=1460 \mathrm{~m} / \mathrm{s}$ for the acoustic medium. The modulus $B_{c}$, thickness $h_{c}$, and loss factor are chosen as $10^{6} \mathrm{~Pa}, 0.01 \mathrm{~m}$, and 0 , respectively. A transverse point force-excitation applied at $(0.06,0.06) \mathrm{m}$ forms one corner of the plate. For all the calculations in the paper, the Fourier series terms used are truncated to $M=N=12$ and $T=S=20$.

3.1. Validation of the Proposed Formulation. In the literature, the sound radiation from a plate with simply supported boundary conditions has been extensively studied. The sound radiation from a simply supported plate that emerged in water with decoupling layer was studied [17], using a model that takes strong coupling between the plate and water into consideration. As previously mentioned, the proposed formulation offers a general method for the calculation of sound radiation from plates with decoupling layer with any boundary conditions. Under the current framework, the classical homogeneous mounting conditions can be simply viewed as the special cases when each elastic restraint is either extremely weak or strong. To compare with the results in [17], the simply supported boundary conditions can be readily simulated by setting the stiffness values of the transverse and rotational spring to infinity and zero, respectively. Figure 2 shows the mean-square velocity of the base plate compared with [17]. From Figure 2, the current results are found to match very well with those in [17].
3.2. Sound Radiation from Plates with Different Boundary Conditions. To study the effects of the mounting conditions on the sound radiation and reduction, different classic mounting conditions are first applied on the edges. The classic mounting conditions, simply supported (S), guided (G), and clamped (C), are studied. In Figures 3 and 4, three types of mounting conditions, SSSS, GGGG, and CCCC, are chosen to calculate the radiated power and power reduction. Similar to the vibration of plate, the radiated sound power and power reduction are different with different mounting conditions applied at the edges. From Figure 3 it is seen that sound power radiated from the guided plate is larger than the situation when the plate is simply supported or clamped at the specific frequencies below $100 \mathrm{~Hz}$. The sound power radiated from the clamped and simply supported plates increases with the increasing of the frequency especially at $10-100 \mathrm{~Hz}$, while the power radiated from the guided plate keeps nearly the same. The peaks on the curves are mainly affected by the natural frequencies of the plates. Figure 4 shows that, at some particular frequencies, sound power reduction is negative, which means that the introduction of decoupling layer increases the sound power radiated into water at those frequencies. The curve values increase with the increasing of the frequency, except some peaks and troughs. Larger sound power reduction is derived for the high frequency since the thickness deformation plays a more important role in isolating the vibration with the increasing of frequency. To reduce the sound power radiation at low frequency, below $100 \mathrm{~Hz}$, some other strategies should be adopted instead of adding decoupling layers to the plate to get a better sound reduction effect.

Next, the sound radiation from plates with elastic boundary condition is studied. To avoid the difficulty of dealing with several variables simultaneously, all four edges are subjected to the same elastic restraint. The stiffnesses of transverse and 


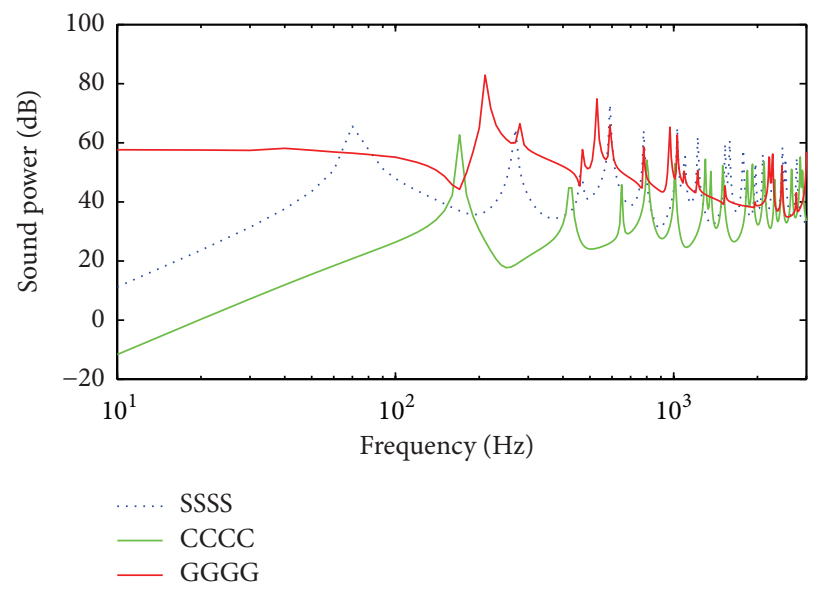

FIGURE 3: Radiated sound power under different mounting conditions (SSSS: simply supported; GGGG: guided; and CCCC: clamped).

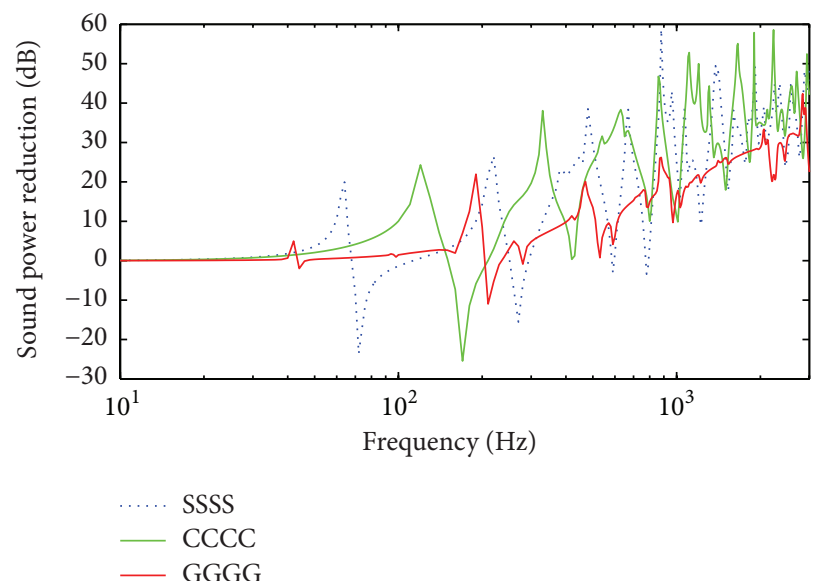

FIGURE 4: Sound reduction provided by the decoupling layer when the plate is under classic mounting boundaries (SSSS: simply supported; GGGG: guided; and CCCC: clamped).

rotational springs take on different values: $K a / D\left(\right.$ and $\left.k a^{3} / D\right)$ $=0.1$ to $10^{6}$. Plotted in Figure 5 is the radiated sound power from the plate restrained by varying stiffnesses under the exciting frequencies of $300 \mathrm{~Hz}$ and $500 \mathrm{~Hz}$. In addition, the sound power reductions are shown in Figure 6. In Figure 5, the dark red points mean that the natural frequencies of the structure match the exciting frequencies, $300 \mathrm{~Hz}$ and $500 \mathrm{~Hz}$. From Figure 6, it can be observed that a better sound reductions are obtained when $K a / D>10^{2}$ and $k a^{3} / D>10^{4}$, which is known as the clamped boundaries. Sound reduction, shown in Figure 6, is negative for some combination of restraints, which means that the plates radiate more power when introducing the decoupling layer with those particular restraints. In the practical design, both sound power radiation and reduction should be taken into consideration to get a better control of sound radiation. Modifying restrained conditions hence represents a viable design option for controlling the sound power radiation with or without the decoupling layers.
3.3. Sound Radiation from Plates with Different Thicknesses of the Decoupling Layer. For the most studies in the literature, the distribution of the decoupling layer is uniform and the thickness of the decoupling layer is kept as a constant along the plate scale. In this case, the sound radiated from the nonuniform distributed decoupling layer is studied, which can be obtained from (6). Two types of thickness distributions are studied: a uniform one, with $h_{c 1}(x, y)=10 \mathrm{~mm}$, and a nonuniform one, with $h_{c 2}(x, y)=10+5^{*} \cos (\pi x / a) \cos (\pi y / b)$ $\mathrm{mm}$, as shown in Figure 7 . In these two cases, the amounts of the decoupling layer adopted are the same. From Figure 7, the nonuniform distribution of decoupling layer is observed to radiate more power than the uniform one at most of the frequency bands. The peak points on both curves keep nearly the same since the changing of decoupling layers at those two situations has no significant influence on the natural frequencies of these structures. For practical application, the uniform distribution of the decoupling layer will generate a better sound reduction effect for most of the situations. 


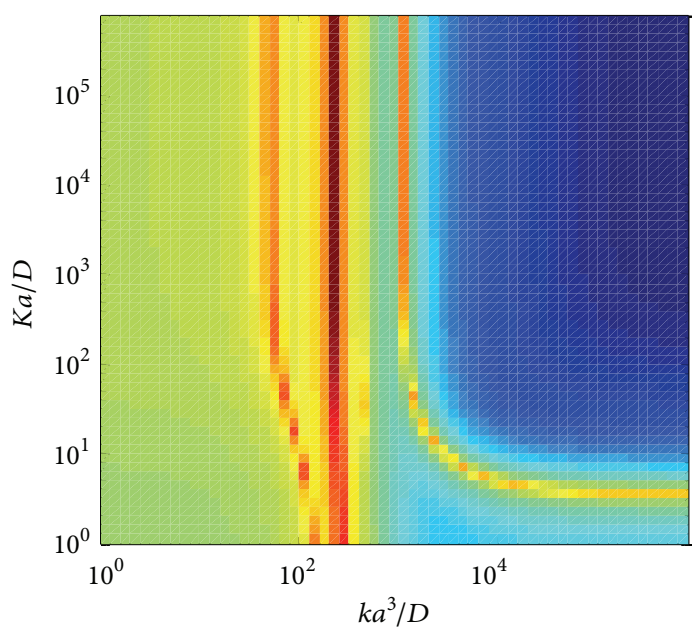

(a)

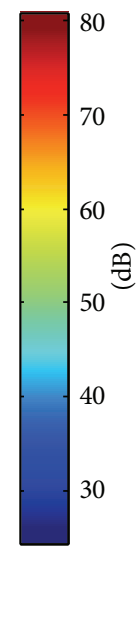

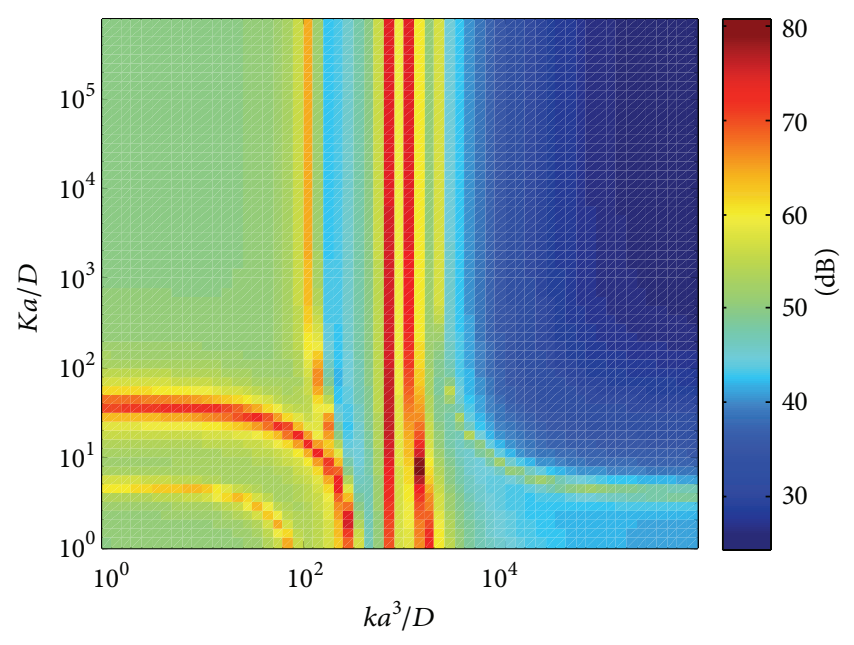

(b)

FIGURE 5: Radiated power with varying restraints against rotation and translation ((a) $300 \mathrm{~Hz}$, (b) $500 \mathrm{~Hz}$.

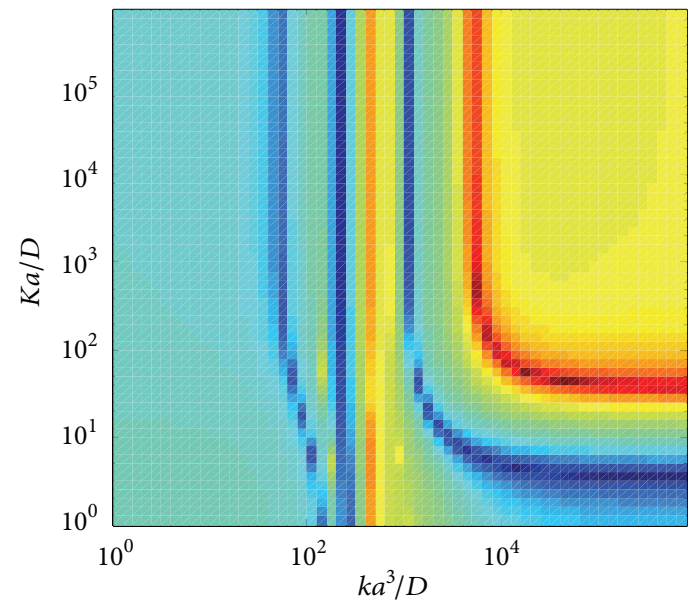

(a)

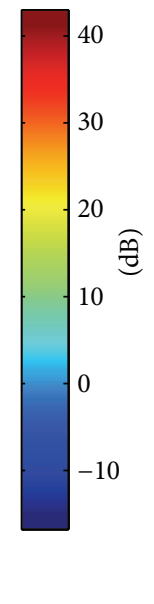

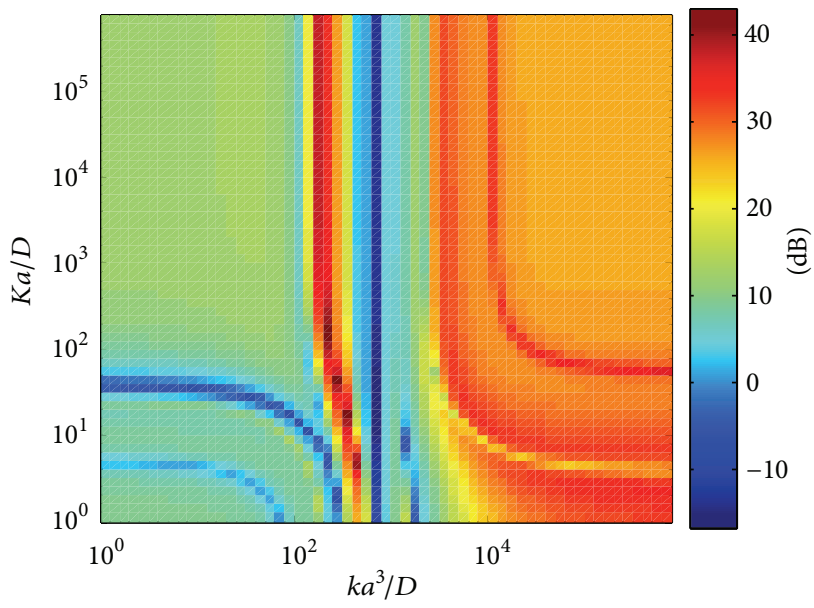

(b)

FIGURE 6: Sound power reductions with varying restraints against rotation and translation ((a) $300 \mathrm{~Hz}$, (b) $500 \mathrm{~Hz}$.

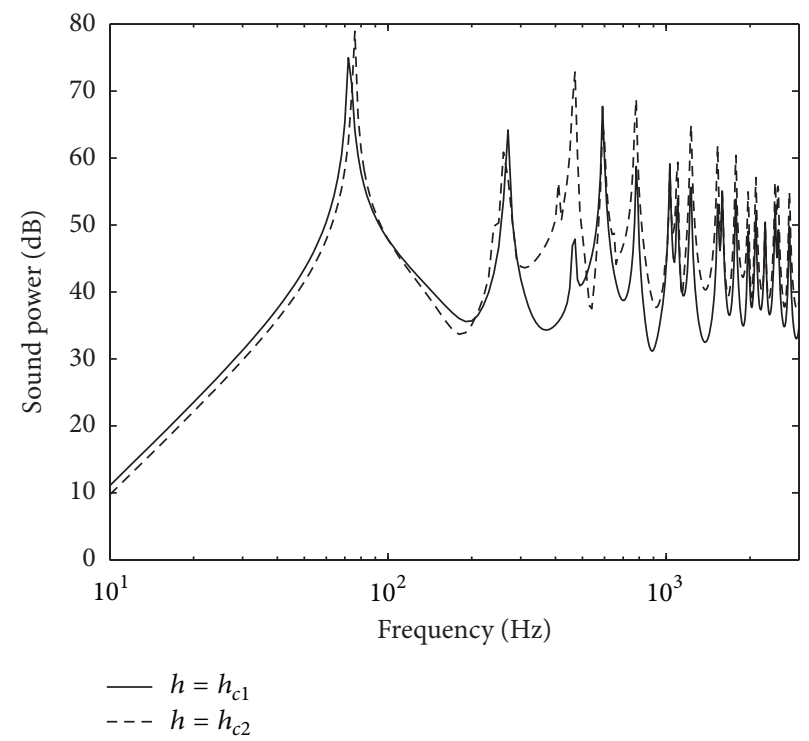

FIGURE 7: Radiated sound powers when the distribution of decoupling layer is uniform and nonuniform. 


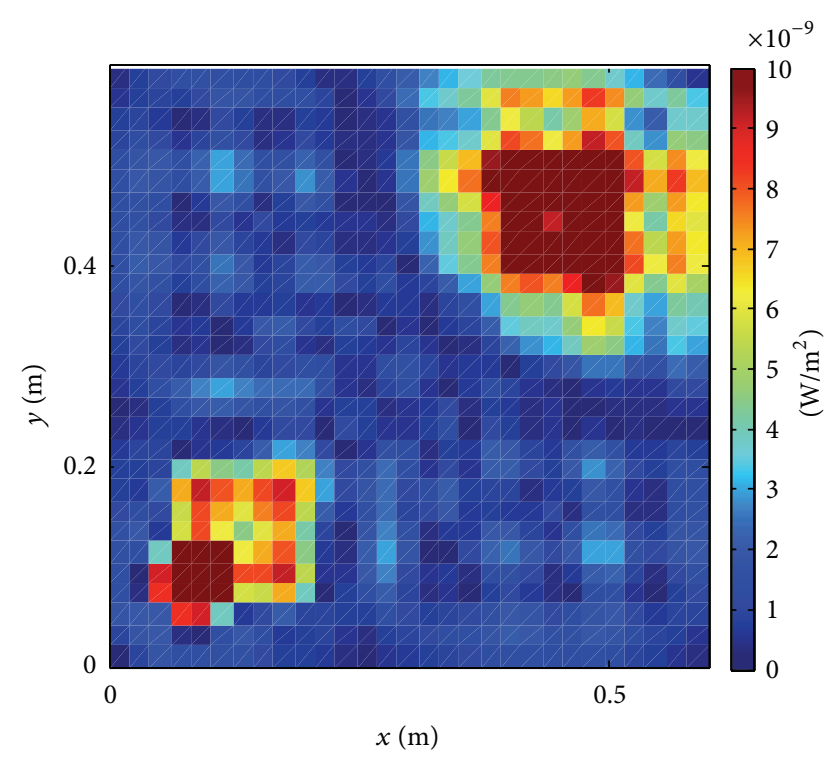

(a)

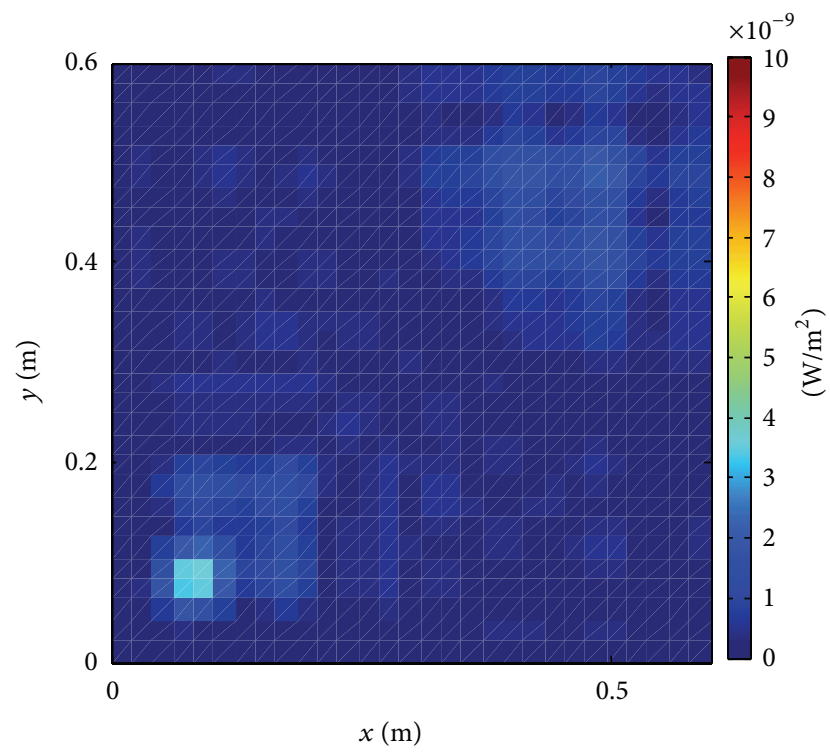

(c)

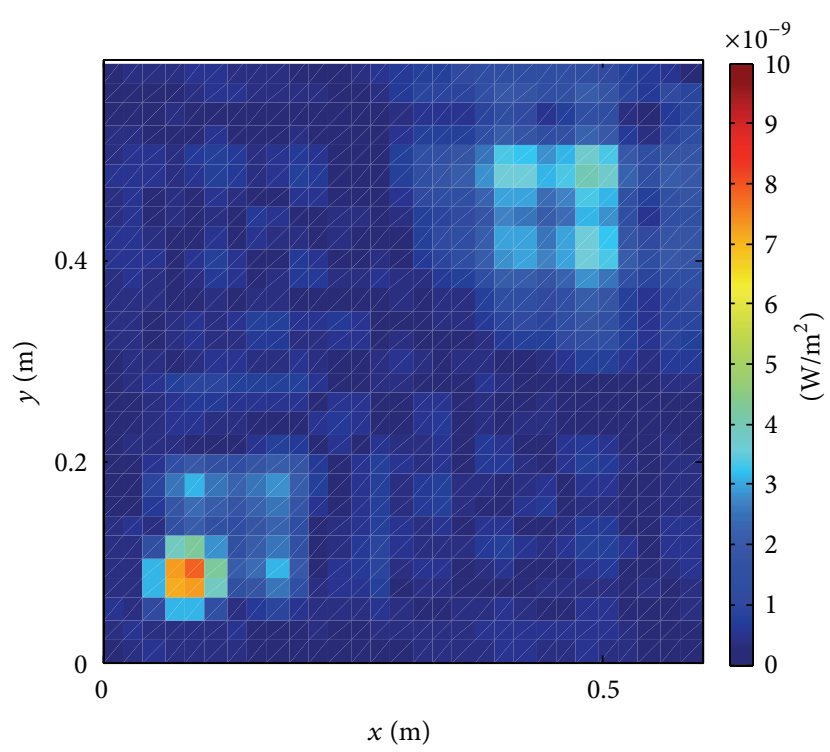

(b)

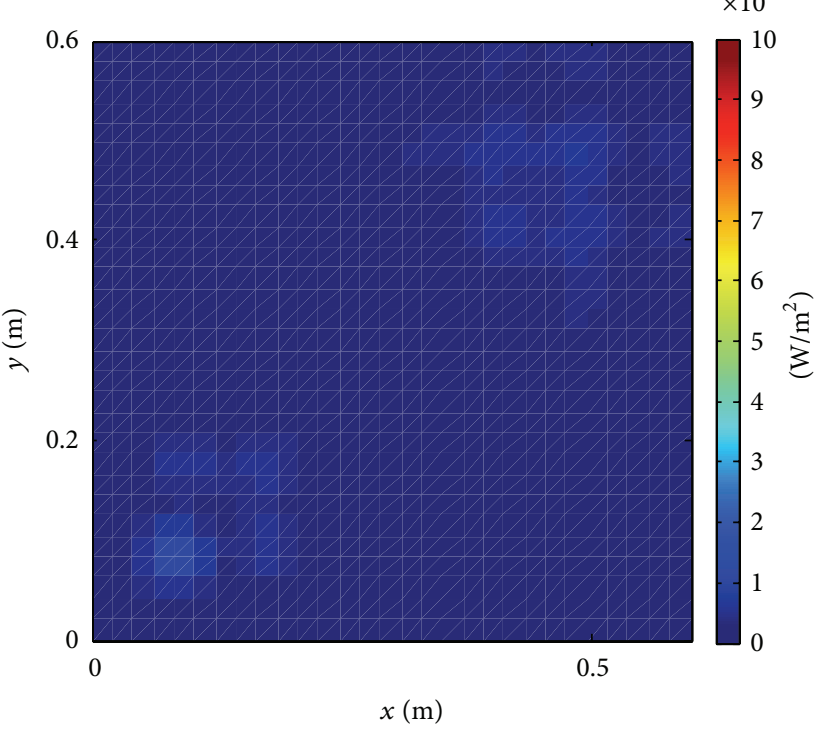

(d)

Figure 8: Sound intensity on the outer surface of the decoupling layer under $400 \mathrm{~Hz}$ excitation frequency ((a) $h=5 \mathrm{~mm}$, (b) $h=10 \mathrm{~mm}$, (c) $h=15 \mathrm{~mm}$, and (d) $h=20 \mathrm{~mm})$.

Finally, the sound intensity distribution on the outer surface of the decoupling layer is analyzed with different decoupling layer thicknesses under the simply supported boundary conditions. Four different thicknesses of the decoupling layer are studied: 5, 10, 15, and $20 \mathrm{~mm}$. Figures 8 and 9 show the sound intensity on the outer surface of the decoupling layer under $400 \mathrm{~Hz}$ and $500 \mathrm{~Hz}$ excitation frequencies, respectively. The sound intensity distributions are mainly affected by the modal shapes at those exciting frequencies. In Figure 8, the dark red point at the lower left corner is the place where the exciting force is applied, and the red point at the top right corner means that the transfer function value is large at this particular frequency. The same phenomenon could also be seen in Figure 9. From these two figures, it can be observed that, with increasing thickness, the sound intensity decreases, and the distribution of sound intensity tends to be much more uniform along the plate scale because of the isolation of the fluid from the base plate. In the extreme situation, the outer surface of the decoupling layer will act like a piston when the decoupling layer is sufficiently thick.

\section{Conclusion}

The sound radiation from an elastically mounted plate covered by a decoupling layer immersed in semi-infinite water has been studied in this paper. The elastic boundary 


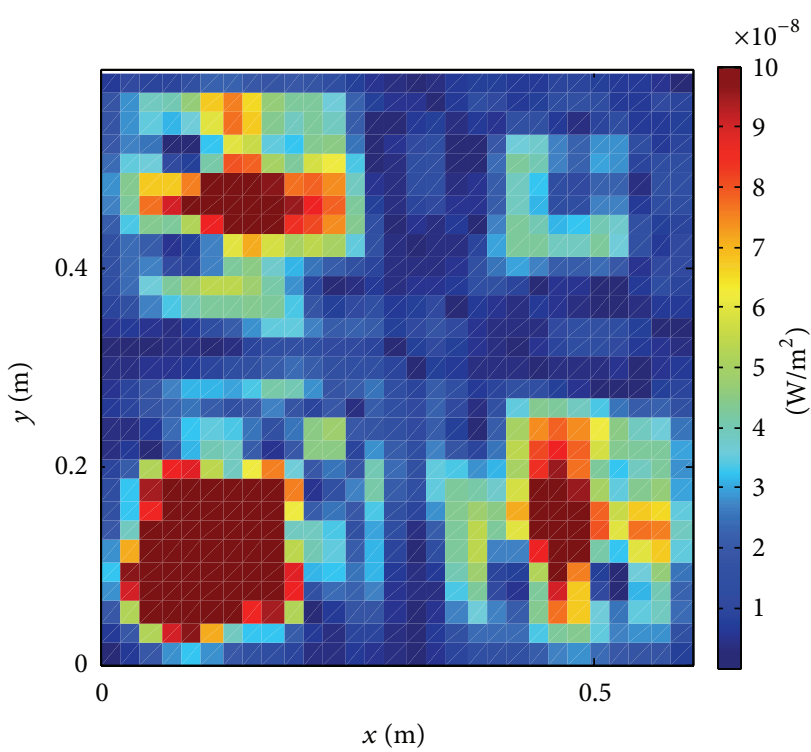

(a)

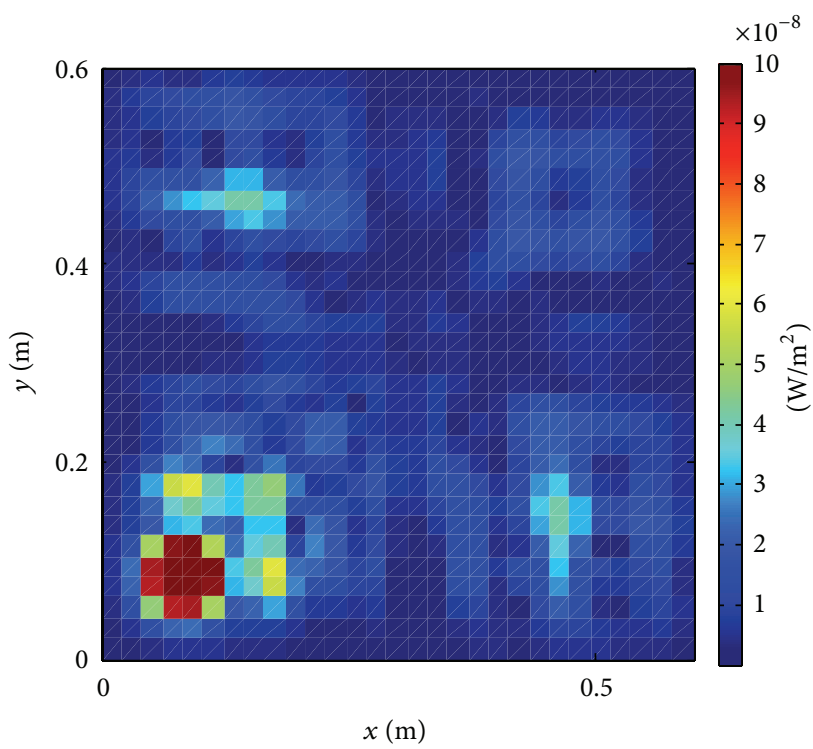

(c)

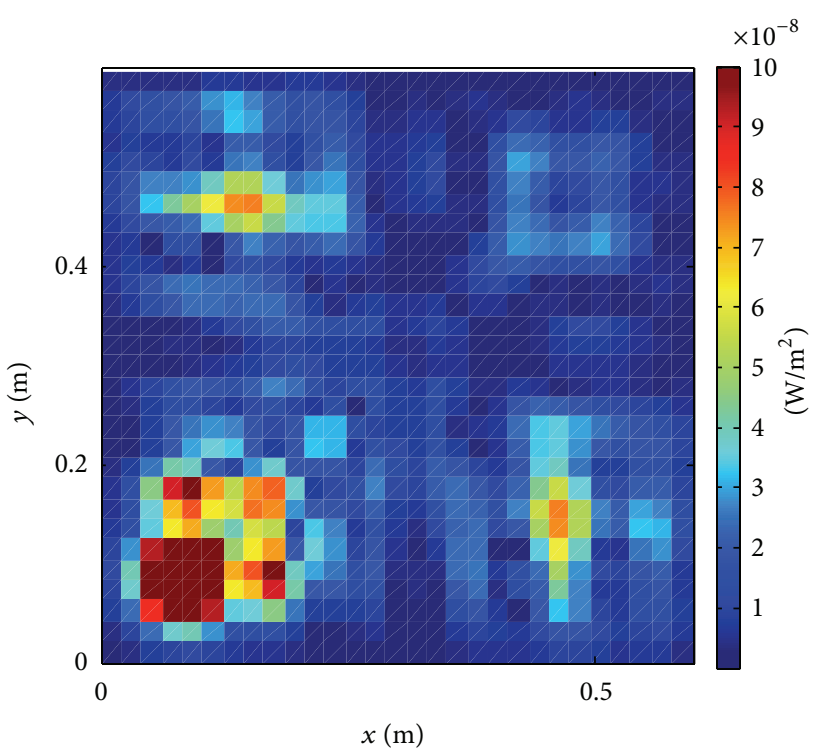

(b)

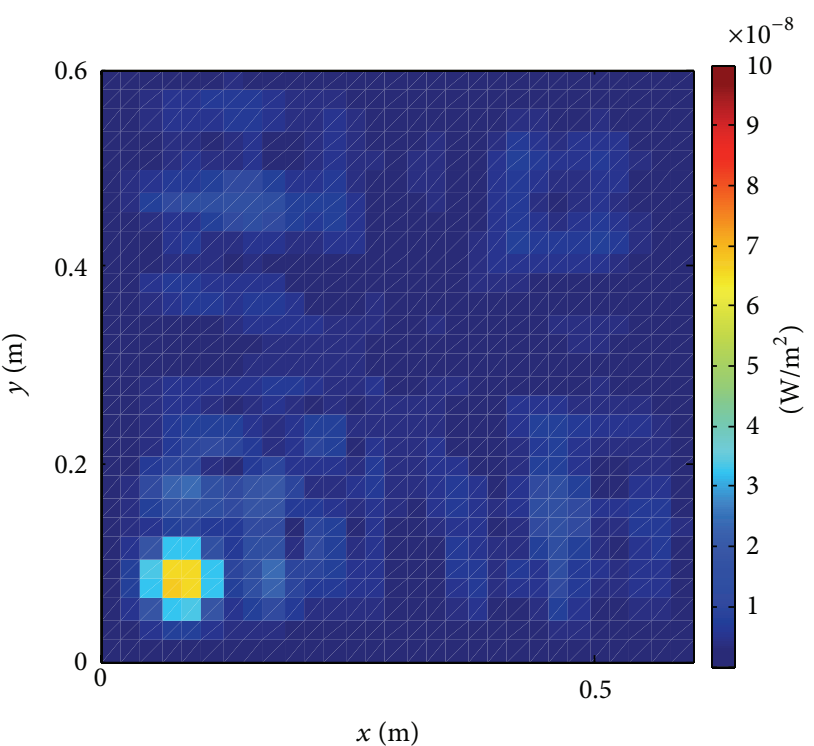

(d)

FIGURE 9: Sound intensity on the outer surface of the decoupling layer under $500 \mathrm{~Hz}$ excitation frequency ((a) $h=5 \mathrm{~mm}$, (b) $h=10 \mathrm{~mm}$, (c) $h=15 \mathrm{~mm}$, and (d) $h=20 \mathrm{~mm}$ ).

condition is realized by applying elastic restraints against both deflection and rotation on the four edges of the rectangular plate. Any uniform or nonuniform boundary condition can be directly taken into account by specifying the stiffness distributions accordingly. A fully vibroacoustic coupling model is established. By analyzing the influence of restrained stiffness, the restraints are found to play very important roles in determining the sound radiation and reduction. The uniform distribution of the decoupling layer has a slightly better sound reduction effect than the nonuniform distribution one when the same amount of decoupling layer is adopted. For some particular combinations of restraints, the introduction of the decoupling layer increases the sound power radiated into water. The sound intensity distribution on the surface of decoupling layer tends to decrease and be much more uniform when the thickness of decoupling layer increases because of the isolation of the fluid from the vibration of the base structure.

\section{Conflict of Interests}

The authors declare that there is no conflict of interests regarding the publication of this paper.

\section{Acknowledgments}

The research work is supported by the National Natural Science Foundation of China (Grant no. 51375104) and 
Heilongjiang Province Funds for Distinguished Young Scientists (Grant no. JC 201405).

\section{References}

[1] D. Feit and J. M. Cuschieri, "Scattering of sound by a fluidloaded plate with a distributed mass inhomogeneity," Journal of the Acoustical Society of America, vol. 99, no. 5, pp. 2686-2700, 1996.

[2] J. M. Cuschieri and D. Feit, "A hybrid numerical and analytical solution for the Green's function of a fluid-loaded elastic plate," Journal of the Acoustical Society of America, vol. 95, no. 4, pp. 1998-2005, 1994.

[3] O. Foin, J. Nicolas, and N. Atalla, "An efficient tool for predicting the structural acoustic and vibration response of sandwich plates in light or heavy fluid," Applied Acoustics, vol. 57, no. 3, pp. 213-242, 1999.

[4] H. Nelisse, O. Beslin, and J. Nicolas, "Fluid-structure coupling for an unbaffled elastic panel immersed in a diffuse field," Journal of Sound and Vibration, vol. 198, no. 4, pp. 485-506, 1996.

[5] J. M. Cuschieri and D. Feit, "Full numerical solution for the farfield and near-field scattering from a fluid-loaded elastic plate with distributed mass or stiffness inhomogeneity," Journal of the Acoustical Society of America, vol. 104, no. 2, pp. 915-925, 1998.

[6] Z. Wang, X. Liang, and G. Liu, "An analytical method for evaluating the dynamic response of plates subjected to underwater shock employing Mindlin plate theory and Laplace transforms," Mathematical Problems in Engineering, vol. 2013, Article ID 803609, 11 pages, 2013.

[7] D. L. Xiang, J. L. Rong, and X. He, "Experimental investigation of dynamic response and deformation of aluminium honeycomb sandwich panels subjected to underwater impulsive loads," Shock and Vibration, vol. 2015, Article ID 650167, 13 pages, 2015.

[8] M. L. Rumerman, "Radiation efficiencies for steel plates in water," Shock and Vibration, vol. 14, no. 2, pp. 81-88, 2007.

[9] S. Chonan and Y. Kugo, "Acoustic characteristics and the design of two-layered soundproof plates," Journal of Sound and Vibration, vol. 129, no. 3, pp. 501-511, 1989.

[10] S. H. Ko, "Reduction of structure-borne noise using an airvoided elastomer," Journal of the Acoustical Society of America, vol. 101, no. 6, pp. 3306-3312, 1997.

[11] R. F. Keltie, "Signal response of elastically coated plates," Journal of the Acoustical Society of America, vol. 103, no. 4, pp. 1855-1863, 1998.

[12] Y. Zhang and J. Pan, "Underwater sound scattering and absorption by a coated infinite plate with a distributed inhomogeneity," Journal of the Acoustical Society of America, vol. 133, no. 4, pp. 2082-2096, 2013.

[13] Y. Zhang and J. Pan, "Underwater sound radiation from an elastically coated plate with a discontinuity introduced by a signal conditioning plate," Journal of the Acoustical Society of America, vol. 133, no. 1, pp. 173-185, 2013.

[14] M. Tao, W. L. Tang, and H. X. Hua, "Noise reduction analysis of an underwater decoupling layer," Journal of Vibration and Acoustics, Transactions of the ASME, vol. 132, no. 6, Article ID 061006, 2010.

[15] D. G. Crighton, "Aspects of the reflexion and free wave properties of a composite panel under fluid loading," Journal of Sound and Vibration, vol. 64, no. 4, pp. 467-474, 1979.
[16] M. R. Garrison, R. N. Miles, J. Q. Sun, and W. Rao, "Random response of a plate partially covered by a constrained layer damper," Journal of Sound and Vibration, vol. 172, no. 2, pp. 231245, 1994.

[17] O. Foin, A. Berry, and J. Szabo, "Acoustic radiation from an elastic baffled rectangular plate covered by a decoupling coating and immersed in a heavy acoustic fluid," Journal of the Acoustical Society of America, vol. 107, no. 5, pp. 2501-2510, 2000.

[18] B. Laulagnet and J. L. Guyader, "Sound radiation from a finite cylindrical shell covered with a compliant layer," Journal of Vibration and Acoustics, vol. 113, no. 2, pp. 267-272, 1991.

[19] B. E. Sandman and J. E. Boisvert, "Simplified structural acoustic characterization of external compliant coatings on submerged surfaces," NUWC Division Newport Technical Digest, 1995.

[20] B. Laulagnet and J. L. Guyader, "Sound radiation from finite cylindrical coated shells, by means of asymptotic expansion of three-dimensional equations for coating," Journal of the Acoustical Society of America, vol. 96, no. 1, pp. 277-286, 1994.

[21] A. K. Noor and W. S. Burton, "Assessment of shear deformation theories for multilayered composite plates," Applied Mechanics Reviews, vol. 42, no. 1, pp. 1-13, 1989.

[22] F. Xiao, Y. Chen, Y. Wang, H. Hua, and D. Zhu, "Experimental research on the dynamic response of floating structures with coatings subjected to underwater explosion," Shock and Vibration, vol. 2014, Article ID 705256, 13 pages, 2014.

[23] L. C. F. Sanches, E. Mesquita, R. Pavanello, and L. Palermo Jr., "Dynamic stationary response of reinforced plates by the boundary element method," Mathematical Problems in Engineering, vol. 2007, Article ID 62157, 17 pages, 2007.

[24] S. Brischetto, E. Carrera, and L. Demasi, "Free vibration of sandwich plates and shells by using zig-zag function," Shock and Vibration, vol. 16, no. 5, pp. 495-503, 2009.

[25] F. Tornabene and A. Ceruti, "Mixed static and dynamic optimization of four-parameter functionally graded completely doubly curved and degenerate shells and panels using GDQ method," Mathematical Problems in Engineering, vol. 2013, Article ID 867079, 33 pages, 2013.

[26] F. G. Leppington, E. G. Broadbent, and K. H. Heron, "Acoustic radiation from rectangular panels with constrained edges," Proceedings of the Royal Society of London, Series A: Mathematical and Physical Sciences, vol. 393, no. 1804, pp. 67-84, 1984.

[27] F. J. Fahy, "Vibration of containing structures by sound in the contained fluid," Journal of Manufacturing Science and Engineering, vol. 91, pp. 939-950, 1969.

[28] O. Beslin and J. Nicolas, "A hierarchical functions set for predicting very high order plate bending modes with any boundary conditions," Journal of Sound and Vibration, vol. 202, no. 5, pp. 633-655, 1997.

[29] H. Nelisse, O. Beslin, and J. Nicolas, "A generalized approach for the acoustic radiation from a baffled or unbaffled plate with arbitrary boundary conditions, immersed in a light or heavy fluid," Journal of Sound and Vibration, vol. 211, no. 2, pp. 207225, 1998.

[30] X. Zhang and W. L. Li, "A unified approach for predicting sound radiation from baffled rectangular plates with arbitrary boundary conditions," Journal of Sound and Vibration, vol. 329, no. 25, pp. 5307-5320, 2010. 


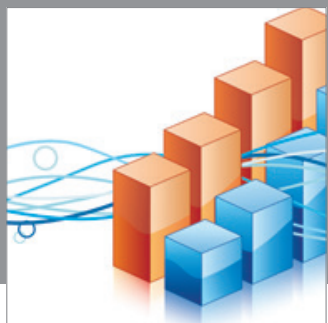

Advances in

Operations Research

mansans

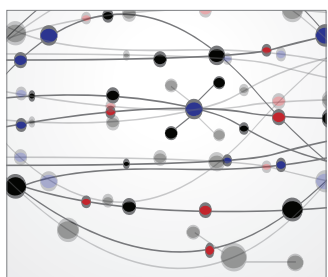

The Scientific World Journal
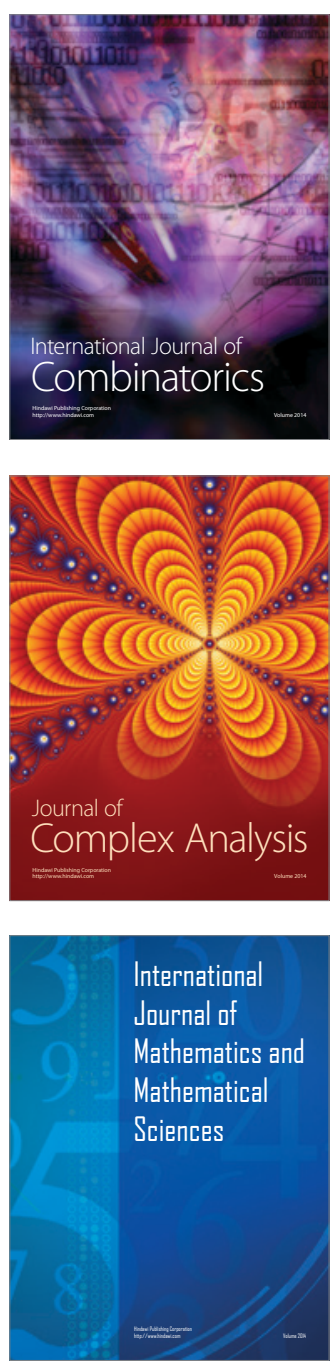
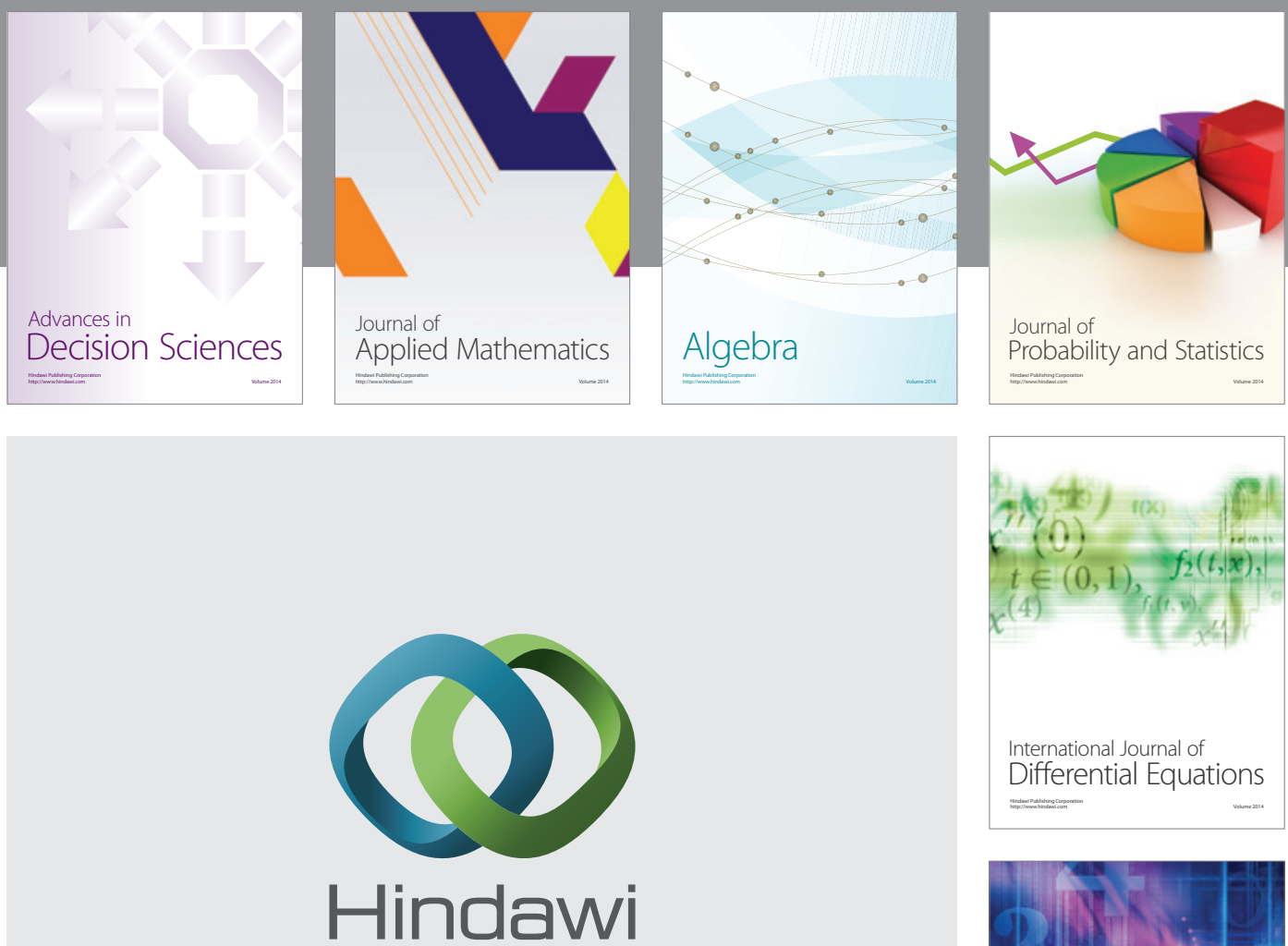

Submit your manuscripts at http://www.hindawi.com
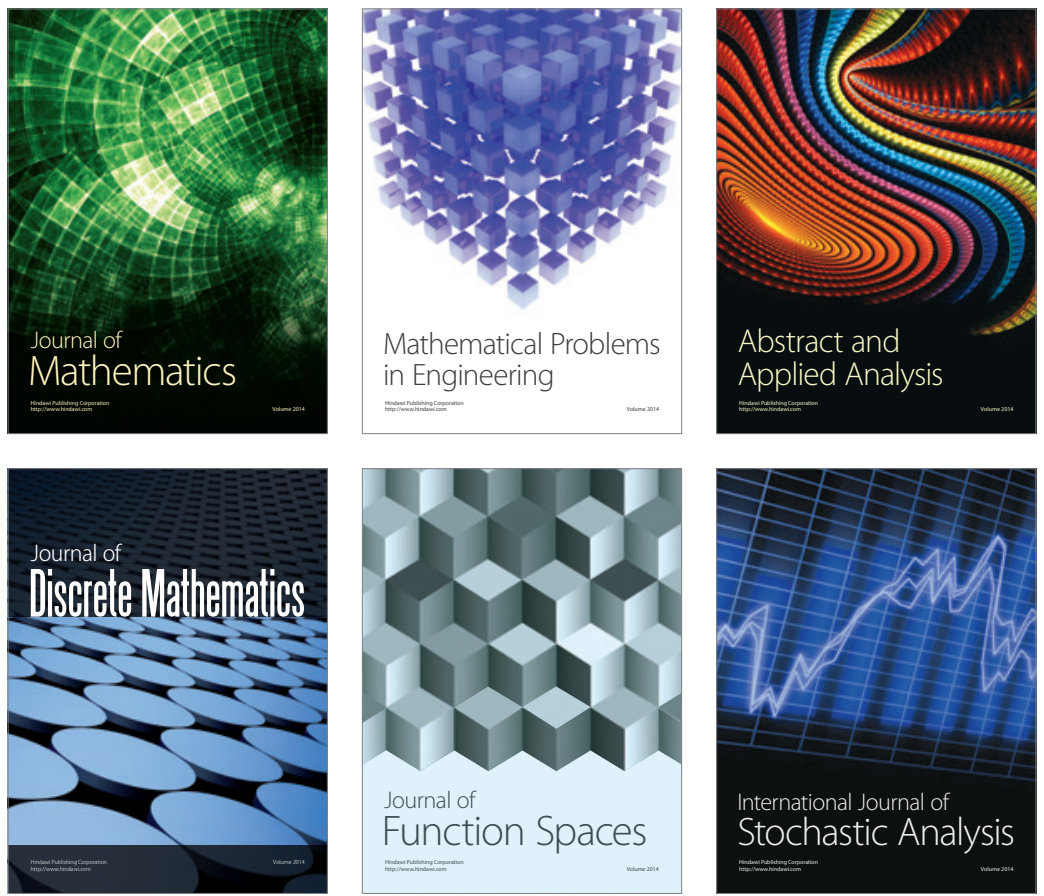

Journal of

Function Spaces

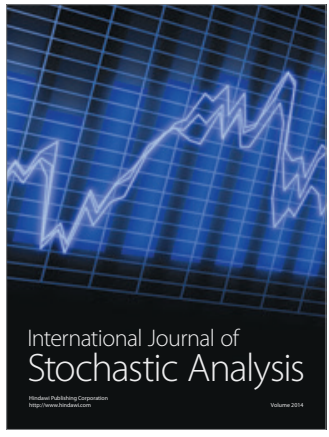

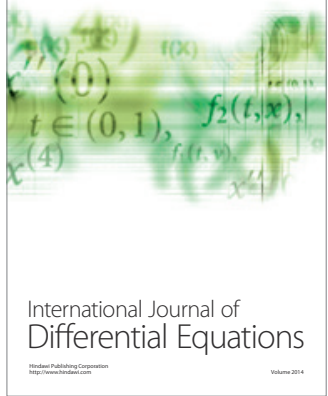
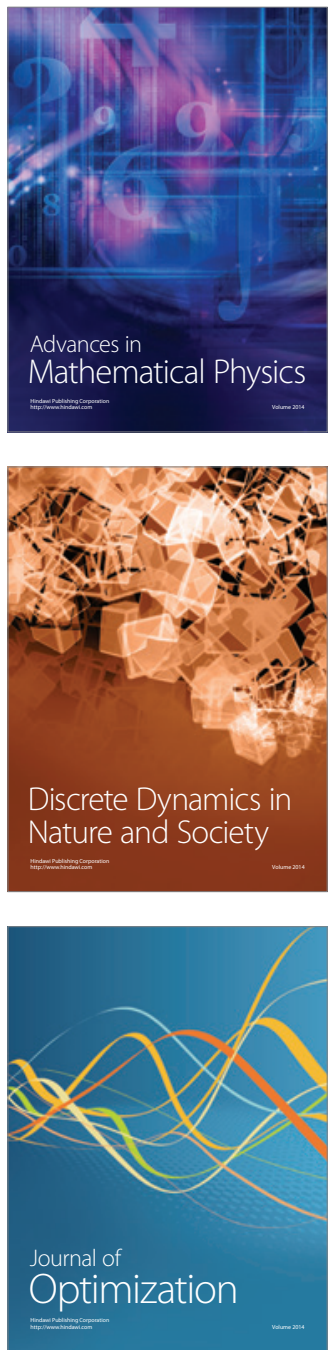